\title{
Vacinação extra-PNV dos 2-4 anos de idade: fatores associados à não realização e perspetiva do pediatra vs médico de família
}

Joana Teixeira, ${ }^{1}$ Maria Miguel Gomes, ${ }^{1}$ Marina Gonçalves, ${ }^{2}$ Ana Gomes ${ }^{2}$

\section{RESUMO}

Objetivos: Pretendemos conhecer os fatores associados à não realização e as recomendações do médico de medicina geral e familiar (MGF) e do pediatra acerca destas vacinas.

Tipo de estudo: observacional, transversal, descritivo.

Local: USF da zona norte do País (Braga - USF de Ruães).

População: Crianças com idades compreendidas entre os 2 e os 4 anos de idade; pediatras e MGF de Braga.

Métodos: Consulta do processo clínico eletrónico da criança juntamente com um questionário realizado por via telefónica, respondido pelos pais ou encarregados de educação, no período de agosto de 2014 a janeiro de 2015. Para avaliação das recomendações dos médicos MGF e pediatra foi aplicado um questionário eletrónico, de resposta online, a médicos de pediatria e MGF. Os dados foram analisados com recurso ao programa Statistical Package for the Social Sciences (SPSS ${ }^{\circledR}$, Chicago, IL, USA), versão 17.0 para Microsoft Windows ${ }^{\circledR}$.

Resultados: Amostra de 180 crianças, dos 2-4 anos de idade. A taxa de cobertura vacinal contra Streptococcus pneumoniae 13-valente foi de $81,7 \%$. Não se encontrou relação estatisticamente significativa entre o desemprego e a não realização das vacinas extra-PNV. Verificamos uma maior vacinação contra Neisseria meningitidis do serogrupo B e contra o vírus da hepatite A (VHA) na ausência de insuficiência económica ( $p$ value de Fisher $=0,008$ e $p=0,046$, respetivamente). Verificou-se uma associação entre a realização da vacina contra o rotavírus e frequência de infantário $(p=0,029)$. Observou-se também uma associação estatisticamente significativa entre as crianças cuja escolaridade do pai ou da mãe era licenciatura/bacharelato e a realização das vacinas contra o rotavírus e anti-VHA. O elemento informador das vacinas extra-PNV foi o médico de família em $46 \%$. A realização de todas as vacinas extra-PNV (exceto gripe sazonal) foi superior nas crianças que eram ou tinham sido seguidas por pediatra.

Quanto ao questionário aplicado aos médicos obtivemos uma amostra total de 30 MGF e 30 pediatras. Revelou que a vacina mais aconselhada em ambos os grupos foi a contra Streptococcus pneumoniae. A ordem de importância atribuída às vacinas extra-PNV foi a mesma entre os dois grupos de profissionais. A principal razão para nem sempre aconselharem a vacina contra Streptococcus pneumoniae foi o preço.

Conclusões: Para a maioria destas vacinas, desemprego e insuficiência económica não são fundamentais na decisão de não vacinar, o que salienta o esforço por parte dos pais em garantir a vacinação. É urgente a tomada de medidas que permitam um acesso mais justo às diferentes opções vacinais.

Palavras-chave: Vacinação; Programa Nacional de Vacinação; Não-cumprimento. 


\section{INTRODUÇÃO}

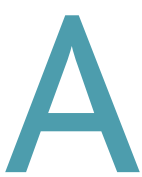

o longo dos tempos foram várias as estratégias desenvolvidas para a proteção das populações contra as doenças infeciosas, mas nenhuma tão eficaz quanto a vacinação. ${ }^{1-2}$ Existem atualmente, além das vacinas contempladas no Programa Nacional deVacinação (PNV), vacinas não incluídas, nomeadamente a vacina contra Streptococcus pneumoniae (não incluída no grupo etário em estudo), contra o rotavírus, contra Neisseria meningitidis do serogrupo $B$, contra a varicela, contra o vírus da hepatite A (VHA), contra a gripe sazonal e contra a Bordetella pertussis..$^{3-4}$ Em 2014, a comissão de vacinas da Sociedade de Infeciologia Pediátrica da Sociedade Portuguesa de Pediatria (SIP-SPP) emitiu um documento com as novas recomendações acerca das vacinas extra$\mathrm{PNV}^{3}$.

A introdução, em 2010, da vacina antipneumocócica 13 valente em Portugal tem permitido a proteção contra um maior número de casos de doença invasiva pneumocócica. ${ }^{5-8}$ Relativamente à vacinação antirrotavírus, estão já licenciadas desde 2006 e 2008 uma vacina pentavalente e uma monovalente, respetivamente, que permitiram reduzir a prevalência e custos associados às gastroenterites e diarreia aguda nas crianças. ${ }^{8-10}$ Mais recentemente começou a ser comercializada a nova vacina contra a Neisseria meningitidis do serogrupo B. ${ }^{11}$

Um dos objetivos inscritos nas orientações técnicas da Direção-Geral da Saúde (DGS) para as consultas de saúde infantil e juvenil é a monitorização e incentivo vacinal. ${ }^{12-14}$ A questão económica como condicionante da vacinação extra-PNV é um fenómeno global, surgindo também como fator limitador da sua prescrição pelos médicos, apesar destes estarem uniformemente convictos da sua eficácia e utilidade. ${ }^{12-13,15}$

Na maioria dos países europeus, dada a importância da proteção conferida pela vacina contra Streptococcus pneumoniae, esta encontra-se incluída no PNV, estando disponível para vacinação gratuita, o que só em 2015 aconteceu em Portugal. ${ }^{3,5} \mathrm{O}$ custo das vacinas extra-PNV não é suportado pelo Sistema Nacional de Saúde.

Em 2010, Rocha et l $^{13}$ descreveram alguns fatores sociodemográficos associados ao não cumprimento da vacina contra Streptococcus pneumoniae 7-valente e contra o rotavírus. Desde então, quer em Portugal quer nos países europeus, não há estudos sobre os fatores associados à não realização destas vacinas quando são suportadas pelo agregado familiar. Assim, os objetivos deste trabalho são o estudo da cobertura vacinal, não apenas da vacina contra Streptococcus pneumoniae e rotavírus, mas de todas as vacinas extra-PNV disponíveis para as crianças até aos quatro anos de idade. Partindo da hipótese de que o acesso a estas vacinas não é igual para todas as crianças portuguesas pelo fator discriminatório económico existente, pretendemos determinar os fatores associados à não realização destas vacinas numa população rural de Braga, alargando o conhecimento a outros ainda não estudados como a existência de desemprego no agregado familiar e a frequência de infantário. Relativamente à vacina contra Streptococcus pneumoniae, este estudo propõe-se avaliar a forma 13-valente. Adicionalmente pretendemos conhecer as recomendações e fontes de informação do médico de família e do pediatra acerca das vacinas extra-PNV.

\section{MÉTODOS}

Estudo observacional, transversal e descritivo, realizado através da consulta do processo clínico eletrónico do doente juntamente com um questionário realizado por via telefónica, respondido pelos pais ou encarregados de educação, que decorreu no período de agosto de 2014 a janeiro de 2015. Para cumprir o último objetivo proposto foi aplicado um questionário eletrónico, anónimo e confidencial, de resposta online, a internos de formação específica e assistentes de pediatria e medicina geral e familiar (MGF). Os questionários foram enviados via correio eletrónico institucional para os pediatras do Hospital de Braga e médicos de MGF do Agrupamento de Centros de Saúde do Cávado I - Braga. No final foram considerados os primeiros 30 questionários de cada grupo profissional.

Considerando que a vacinação extra-PNV, de acordo com as recomendações dos fabricantes, fica completa até aos quatro anos de idade, a nossa amostra foi constituída pela totalidade das crianças inscritas numa USF da zona norte do País (Braga-USF de Ruães), com idades compreendidas entre os dois e os quatro anos de idade.

Critérios de inclusão: crianças com idade com- 
preendida entre os dois e os quatro anos de idade e inscrição na USF de Ruães. Critérios de exclusão: não preencher os critérios de inclusão, inscrição posterior à data de vacinação, não compreensão de língua portuguesa, incapacidade de ouvir ou de falar e recusa em participar no estudo.

A fonte de recrutamento utilizada para a obtenção da amostra foi o programa MIM@UF (Módulo de Informação e Monitorização das Unidades Funcionais). Para a obtenção de dados relativamente à inoculação de vacinas nas crianças e características sociodemográficas do agregado familiar foram utilizados o programa informático Sistema de Apoio ao Médico (SAM®, versão 13.2) e informação verbal fornecida pelos pais/encarregados de educação (resposta a questionário telefónico).

A insuficiência económica, no ano vigente à data de realização do estudo (2014), foi definida como rendimento médio mensal per capita igual ou inferior a 628,83 euros.

O esquema vacinal foi categorizado em: esquema completo, se a criança tivesse efetuado o esquema recomendado pelo fabricante da vacina; incompleto, se a criança tivesse iniciado, mas não completado o esquema; não realizado, se a criança não tivesse nenhuma dose da vacina. No caso específico da vacina da gripe sazonal apenas foi registado se a criança já tinha ou não realizado a vacina alguma vez. Quanto às vacinas contra a varicela e VHA, o esquema foi categorizado em: esquema completo, uma dose ou não realizado.

Obtivemos autorização da comissão de ética da Administração Regional de Saúde do Norte.

Os dados foram introduzidos e analisados com recurso ao programa Statistical Package for the Social Sciences (SPSS $\AA$, Chicago, IL, USA), versão 17.0 para Microsoft Windows ${ }^{\circledR}$. As variáveis foram estratificadas de acordo com a realização ou não das vacinas extraPNV disponíveis para vacinação neste grupo etário. Os testes estatísticos utilizados foram o teste Qui Quadrado (X2) e o teste exato de Fisher para comparação entre as variáveis categóricas. Foram consideradas diferenças estatisticamente significativas para um valor de prova $(p)<0,05$.

\section{RESULTADOS}

A análise estatística englobou 180 crianças; sete fo- ram excluídas da análise dos fatores associados à não realização das vacinas extra-PNV por impossibilidade de contacto telefónico. Aproximadamente um terço das famílias apresentava insuficiência económica. As características gerais da amostra estão descritas no quadro I. Verificou-se que $53 \%$ das crianças eram ou já tinham sido acompanhadas por pediatra; $87 \%$ das crianças frequentavam creche ou infantário. A cobertura vacinal extra-PNV para cada uma das vacinas disponíveis nesta faixa etária encontra-se descrita no quadro II. A taxa de cobertura vacinal contra Streptococcus pneumoniae 13 -valente foi a mais elevada com $81,7 \%$.

Relativamente à análise dos fatores associados à não realização das vacinas extra-PNV, não encontramos relação estatisticamente significativa entre a variável desemprego em pelo menos um dos progenitores e a não realização das vacinas extra-PNV. No caso da variável insuficiência económica verificamos que há uma maior vacinação contra Neisseria meningitidis do serogrupo B e contra VHA na ausência de insuficiência económica ( $p$ value Fisher $=0,008$ e $p=0,046$, respetivamente) (quadro III).

Verificou-se uma relação estatisticamente significativa entre administração da vacina contra o rotavírus e a frequência de infantário com um valor de $p=0,029$ (quadro IV). O mesmo não aconteceu para as restantes vacinas extra-PNV.

Quanto à escolaridade observou-se uma associação significativa entre as crianças cuja escolaridade do pai ou da mãe era licenciatura/bacharelato e a realização das vacinas extra-PNV contra o rotavírus e anti-VHA (quadro V).

O elemento informador das vacinas extra-PNV foi o médico de família em $46 \%$ das crianças; em $33,7 \%$ os pais alegaram ter sido o pediatra e em $17,4 \%$ referiram que tanto o pediatra como o médico de família abordaram a questão. $\operatorname{Em} 2,5 \%(n=4)$, os pais desconheciam a existência de vacinas extra-PNV, tratando-se de crianças não vacinadas. A realização de todas as vacinas extra-PNV disponíveis nesta faixa etária foi superior nas crianças que eram ou tinham sido seguidas por pediatra, com exceção da vacina contra a gripe sazonal (quadro IV).

No que respeita aos resultados referentes às recomendações e principais fontes de informação do MGF e do pediatra acerca da vacinação extra-PNV obtive- 
mos uma amostra total de 30 MGF e 30 pediatras, com uma média de 7,3 e 8,2 anos de prática clínica, respetivamente. A ordem de importância atribuída às vacinas extra-PNV disponíveis nesta faixa etária foi maioritariamente a mesma entre os dois grupos de profissionais: vacina contra Streptococcus pneumoniae, contra a Neisseria meningitidis serogrupo B, contra o rotavírus, contra a gripe sazonal, contra oVHA e contra a varicela.

Quanto às principais fontes de informação utilizadas, no grupo dos pediatras foram a Sociedade Portuguesa de Pediatria (SPP) (23/30), literatura/revistas médicas (20/30) e a DireçãoGeral da Saúde (DGS) (18/30). No grupo dos MGF foram a DGS (21/30), literatura/revistas médicas (20/30) e a SPP (19/30). A maior discrepância verificou-se no uso da internet e da indústria farmacêutica como fontes de informação entre pediatras e MGF: 12/30 vs 6/30 em relação à indústria farmacêutica e $1 / 30$ vs $6 / 30$ no caso da internet, respetivamente.

Relativamente ao aconselhamento de cada uma das vacinas extra-PNV, inquirimos os profissionais sobre o número médio de crianças, por cada 100 observadas, a quem recomendavam a realização de cada uma das referidas vacinas. No geral obtivemos taxas de aconselhamento superiores no grupo dos pediatras (quadro VI), sendo que em ambos os grupos as taxas obtidas para a vacina contra Streptococcus pneumoniae 13 valente foram superiores a $90 \%$ das crianças observadas (94\% MGF e $96 \%$ pediatras).

Foram também estudadas as principais razões que levavam médicos a nem sempre aconselharem as diferentes vacinas extra-PNV. O preço foi a principal razão invocada para nem sempre aconselharem as vacinas contra Streptococcus pneumoniae, antirrotavírus e contra Neisseria meningitis do serogrupo B. O facto de não ser considerada uma vacina adequada a todas as crianças foi motivo para os profissionais nem sempre recomendarem as vacinas antirrotavírus, anti-VHA e contra a gripe sazonal. O facto de ser uma vacina controversa foi o moti-

\begin{tabular}{|c|c|c|}
\hline & $n=180$ & $\%$ \\
\hline \multicolumn{3}{|l|}{ Sexo } \\
\hline Feminino & 91 & 50,6 \\
\hline Masculino & 89 & 49,4 \\
\hline \multicolumn{3}{|l|}{ Idade } \\
\hline 2 & 62 & 34,4 \\
\hline 3 & 61 & 33,9 \\
\hline 4 & 57 & 31,7 \\
\hline \multicolumn{3}{|l|}{ Insuficiência económica } \\
\hline Sim & 66 & 36,7 \\
\hline \multirow[t]{2}{*}{ Não } & 114 & 63,3 \\
\hline & $n=173$ & $\%$ \\
\hline \multicolumn{3}{|c|}{ N. ${ }^{\circ}$ crianças do agregado familiar } \\
\hline 1 & 69 & 39,9 \\
\hline 2 & 93 & 53,8 \\
\hline 3 & 10 & 5,8 \\
\hline${ }^{2}$ & 1 & 0,6 \\
\hline \multicolumn{3}{|l|}{ Escolaridade Mãe } \\
\hline Licenciatura/Bacharelato & 43 & 24,9 \\
\hline Ensino secundário & 59 & 34,1 \\
\hline 9. ${ }^{\circ}$ Ano & 47 & 27,2 \\
\hline $6 .^{\circ}$ Ano & 24 & 13,9 \\
\hline \multicolumn{3}{|l|}{ Escolaridade Pai } \\
\hline Licenciatura/Bacharelato & 32 & 18,5 \\
\hline Ensino secundário & 35 & 20,2 \\
\hline 9. Ano & 58 & 33,5 \\
\hline $6 .^{\circ}$ Ano & 48 & 27,7 \\
\hline \multicolumn{3}{|l|}{ Desemprego Pais } \\
\hline Os dois desempregados & 6 & 3,5 \\
\hline Um desempregado & 61 & 35,3 \\
\hline Nenhum & 105 & 60,7 \\
\hline Sem informação & 1 & 0,6 \\
\hline \multicolumn{3}{|l|}{ Tem pediatra assistente? } \\
\hline Sim & 66 & 38,2 \\
\hline Não & 81 & 46,8 \\
\hline Já teve & 26 & 15,0 \\
\hline \multicolumn{3}{|c|}{ Frequenta infantário/creche? } \\
\hline $\operatorname{Sim}$ & 150 & 86,7 \\
\hline \multirow[t]{2}{*}{ Não } & 23 & 13,3 \\
\hline & Média & Desvio-padrão \\
\hline Idade materna (anos) & 34,8 & 4,8 \\
\hline Idade paterna (anos) & 36,8 & 5,5 \\
\hline
\end{tabular}




\begin{tabular}{|c|c|c|}
\hline Vacinas extra-PNV & $n=180$ & $\%$ \\
\hline \multicolumn{3}{|c|}{ Vacina contra Streptococcus pneumoniae } \\
\hline Completo (esquema $2+1$ ) & $147(10)$ & $81,7(5,6)$ \\
\hline Incompleto (falta dose de reforço) & $20(13)$ & $11,1(7,2)$ \\
\hline Não realizado & 13 & 7,2 \\
\hline \multicolumn{3}{|l|}{ Vacina contra o rotavírus } \\
\hline Completo & 110 & 61,1 \\
\hline Incompleto & 8 & 4,4 \\
\hline Não realizado & 62 & 34,4 \\
\hline \multicolumn{3}{|l|}{ Vacina contra vírus da hepatite $\mathrm{A}$} \\
\hline Completo & 17 & 9,4 \\
\hline 1 dose & 9 & 5,0 \\
\hline Não realizado & 154 & 85,6 \\
\hline \multicolumn{3}{|l|}{ Vacina contra a varicela } \\
\hline Completo & 2 & 1,1 \\
\hline 1 dose & 9 & 5,0 \\
\hline Não realizado & 169 & 93,9 \\
\hline \multicolumn{3}{|c|}{ Vacina contra Neisseria meningitidis do serogrupo B } \\
\hline Completo & 6 & 3,3 \\
\hline 1 dose & 5 & 2,8 \\
\hline Não realizado & 169 & 93,9 \\
\hline \multicolumn{3}{|l|}{ Vacina da gripe sazonal } \\
\hline Sim & 20 & 11,1 \\
\hline Não & 160 & 88,9 \\
\hline
\end{tabular}

vo invocado para nem sempre sugerirem as vacinas contra Neisseria meningitis do serogrupo B e varicela. O risco de efeitos secundários foi a razão escolhida para nem sempre aconselharem a vacina contra Neisseria meningitis do serogrupo B. Ser uma doença benigna que não justifica a vacina foi a razão referida no caso das vacinas contra VHA e gripe sazonal. O facto de observarem a criança pela primeira vez já fora do grupo etário foi razão referida por 12 dos 30 pediatras para nem sempre recomendarem a vacina contra o rotavírus.

\section{DISCUSSÃO}

A amostra estudada apresentava uma elevada cobertura vacinal contra Streptococcus pneumoniae, o que nos levanta a questão de qual será atualmente, em Portugal, a taxa de cobertura vacinal. Em Portugal, a doença invasiva pneumocócica (DIP) pediátrica não é de declaração obrigatória; no entanto, são vários os estudos que têm vindo a ser realizados pela Sociedade de Infeciologia Pediátrica no sentido de obter conhecimento sobre a incidência, morbilidade e mortalidade da DIP no nosso país. De acordo com o que tem vindo a ser descrito noutros estudos, a taxa de cobertura vacinal tende a ser alta., ${ }^{5,14,16}$ No entanto, uma medida eficaz seria o apelo à realização da dose de reforço que permitiria aumentar a cobertura vacinal, na amostra estudada, de $81,7 \%$ para $89 \%$.

Importa enfatizar que, para a maioria das vacinas extra-PNV, tanto o desemprego como a insuficiência económica não foram fatores fundamentais na decisão de não vacinar as crianças.

Tal pode estar relacionado com um maior esforço por parte dos pais e familiares em garantir a vacinação das crianças, sobretudo no que concerne à vacina contra Streptococcus pneumoniae 13 valente e contra o rotavírus. Apesar das dificuldades económicas de uma população maioritariamente rural, a taxa de cobertura vacinal para ambas era elevada, mesmo na presença de desemprego e de insuficiência económica. Contudo, a amostra de 180 crianças e o contexto em que o estudo foi realizado (limitado a uma USF do distrito de Braga) constituem fatores limitadores desta conclusão, nomeadamente extrapolação nacional dos resultados.

De referir também que, sendo o infantário uma fonte muito provável de doença por rotavírus nas crianças, verificamos uma correlação fortemente positiva entre frequentar infantário/creche e vacinação contra rotavírus comparativamente às crianças que ficam ao cuidado de um familiar. Este facto estará muito provavelmente relacionado com a maior transmissão deste vírus nos infantários/creches e com o consequente aumento no absentismo laboral dos pais no caso de doença por rotavírus. A maior escolaridade dos pais, associada provavelmente a um maior acesso à informação disponível, foi também um fator preponderante na decisão de vacinar. 


\begin{tabular}{|c|c|c|c|c|c|c|c|c|c|c|c|}
\hline & & \multicolumn{4}{|c|}{ Insuficiência económica } & \multirow[b]{3}{*}{$P$} & \multirow{2}{*}{\multicolumn{2}{|c|}{ Desemprego }} & \multirow{2}{*}{\multicolumn{2}{|c|}{$\begin{array}{c}\text { Nenhum } \\
\text { progenitor } \\
\text { desempregado }\end{array}$}} & \multirow[b]{3}{*}{$P$} \\
\hline & & \multicolumn{2}{|c|}{ Sim } & \multicolumn{2}{|c|}{ Não } & & & & & & \\
\hline & & $n$ & $\%$ & $n$ & $\%$ & & $n$ & $\%$ & $n$ & $\%$ & \\
\hline $\begin{array}{l}\text { Vacina contra } \\
\text { Streptococcus } \\
\text { pneumoniae }\end{array}$ & $\begin{array}{l}\text { Completo/ } \\
\text { Incompleto } \\
\text { Não realizado }\end{array}$ & $\begin{array}{r}48 \\
7\end{array}$ & $\begin{array}{l}(87,3) \\
(12,7)\end{array}$ & $\begin{array}{r}89 \\
6\end{array}$ & $\begin{array}{r}(93,7) \\
(6,3)\end{array}$ & $0,230^{*}$ & 6 & $\begin{array}{l}(89,1) \\
(10,9)\end{array}$ & $\begin{array}{r}84 \\
5\end{array}$ & $\begin{array}{r}(94,4) \\
(5,6)\end{array}$ & $0,334^{*}$ \\
\hline $\begin{array}{l}\text { Vacina contra } \\
\text { o rotavírus }\end{array}$ & $\begin{array}{l}\text { Completo/ } \\
\text { Incompleto } \\
\text { Não realizado }\end{array}$ & $\begin{array}{l}38 \\
28\end{array}$ & $\begin{array}{l}(57,6) \\
(42,4)\end{array}$ & $\begin{array}{l}80 \\
34\end{array}$ & $\begin{array}{l}(70,2) \\
(29,8)\end{array}$ & 0,086 & $\begin{array}{l}41 \\
26\end{array}$ & $\begin{array}{l}(61,2) \\
(38,8)\end{array}$ & $\begin{array}{l}73 \\
32\end{array}$ & $\begin{array}{l}(69,5) \\
(30,5)\end{array}$ & 0,260 \\
\hline $\begin{array}{l}\text { Vacina contra } \\
\text { Neisseria } \\
\text { meningitidis } \\
\text { serogrupo B }\end{array}$ & $\begin{array}{l}\text { Completo/ } \\
1 \text { dose } \\
\text { Não } \\
\text { realizado }\end{array}$ & $\begin{array}{r}0 \\
66\end{array}$ & $\begin{array}{r}(0) \\
(100,0)\end{array}$ & $\begin{array}{r}11 \\
103\end{array}$ & $\begin{array}{r}(9,6) \\
(90,4)\end{array}$ & $0,008^{*}$ & 64 & $\begin{array}{r}(4,5) \\
(95,5)\end{array}$ & $\begin{array}{r}8 \\
97\end{array}$ & $\begin{array}{r}(7,6) \\
(92,4)\end{array}$ & $0,532 *$ \\
\hline $\begin{array}{l}\text { Vacina contra } \\
\text { vírus da } \\
\text { Hepatite A }\end{array}$ & $\begin{array}{l}\text { Completo/ } \\
1 \text { dose } \\
\text { Não realizado }\end{array}$ & $\begin{array}{r}5 \\
61\end{array}$ & $\begin{array}{r}(7,6) \\
(92,4)\end{array}$ & $\begin{array}{l}21 \\
93\end{array}$ & $\begin{array}{l}(18,4) \\
(81,6)\end{array}$ & 0,046 & 8 & $\begin{array}{l}(11,9) \\
(88,1)\end{array}$ & $\begin{array}{l}17 \\
88\end{array}$ & $\begin{array}{l}(16,2) \\
(83,8)\end{array}$ & 0,441 \\
\hline $\begin{array}{l}\text { Vacina contra } \\
\text { a varicela }\end{array}$ & $\begin{array}{l}\text { Completo/ } \\
1 \text { dose } \\
\text { Não realizado }\end{array}$ & $\begin{array}{r}2 \\
64\end{array}$ & $\begin{array}{r}(3,0) \\
(97,0)\end{array}$ & $\begin{array}{r}9 \\
105\end{array}$ & $\begin{array}{r}(7,9) \\
(92,1)\end{array}$ & $0,333^{*}$ & 3 & $\begin{array}{r}(4,5) \\
(95,5)\end{array}$ & $\begin{array}{r}7 \\
98\end{array}$ & $\begin{array}{r}(6,7) \\
(93,3)\end{array}$ & $0,742^{*}$ \\
\hline $\begin{array}{l}\text { Vacina da } \\
\text { gripe sazonal }\end{array}$ & $\begin{array}{l}\text { Sim } \\
\text { Não }\end{array}$ & $\begin{array}{r}5 \\
61\end{array}$ & $\begin{array}{r}(7,6) \\
(92,4)\end{array}$ & $\begin{array}{l}15 \\
99\end{array}$ & $\begin{array}{l}(13,2) \\
(86,8)\end{array}$ & 0,251 & $\begin{array}{r}9 \\
58\end{array}$ & $\begin{array}{l}(13,4) \\
(86,6)\end{array}$ & $\begin{array}{l}11 \\
94\end{array}$ & $\begin{array}{l}(10,5) \\
(89,5)\end{array}$ & 0,628 \\
\hline
\end{tabular}

*Resultado do teste exato de Fisher.

Relativamente à vacina contra Neisseria meningitidis do serogrupo $\mathrm{B}$, uma vacina que à data do estudo era muito recente em Portugal, apenas pudemos verificar que começa já a ser utilizada, dado que na amostra de 180 crianças seis, neste grupo etário, tinham já vacinação completa. Deste modo, podemos questionar: qual será a cobertura vacinal abaixo dos dois anos? Trata-se de uma vacina muito recente e com um preço de mercado elevado para a maioria das famílias, como verificamos pelo facto de a maior percentagem de vacinação contra Neisseria meningitidis do serogrupo B se verificar nas famílias sem insuficiência económica e com menor número de crianças a constituir o agregado familiar. Estaremos nós perante uma vacina disponível para minorias? Hoje em dia defende-se já em alguns países europeus que deveria ser integrada no PNV dadas as vantagens associadas à sua proteção. ${ }^{17} \mathrm{~A}$ doença meningocócica pelo serogrupo B é a mais prevalen- te na Europa. ${ }^{11}$

A maioria das crianças da nossa amostra, sendo saudáveis, não realiza as vacinas contra o vírus da hepatite A, varicela e gripe sazonal. No caso da vacina contra a varicela, sabe-se que não é previsível que esta vacina permita erradicar a doença e, no caso específico de Portugal, não existem muitos dados acerca da hospitalização, complicações e mortalidade relacionadas com o vírus da varicela zooster, o que pode contribuir também para a sua menor recomendação. ${ }^{18}$

Foi possível constatar uma relação positiva entre o seguimento por pediatra (em contexto público ou privado) e um maior índice de vacinação extra-PNV (exceto vacina da gripe sazonal), o que vai ao encontro das respostas obtidas no grupo dos pediatras, com percentagens de aconselhamento superiores aos médicos de MGF. No caso da vacina contra a gripe sazonal, importa salientar a existência de um possível viés, uma vez 


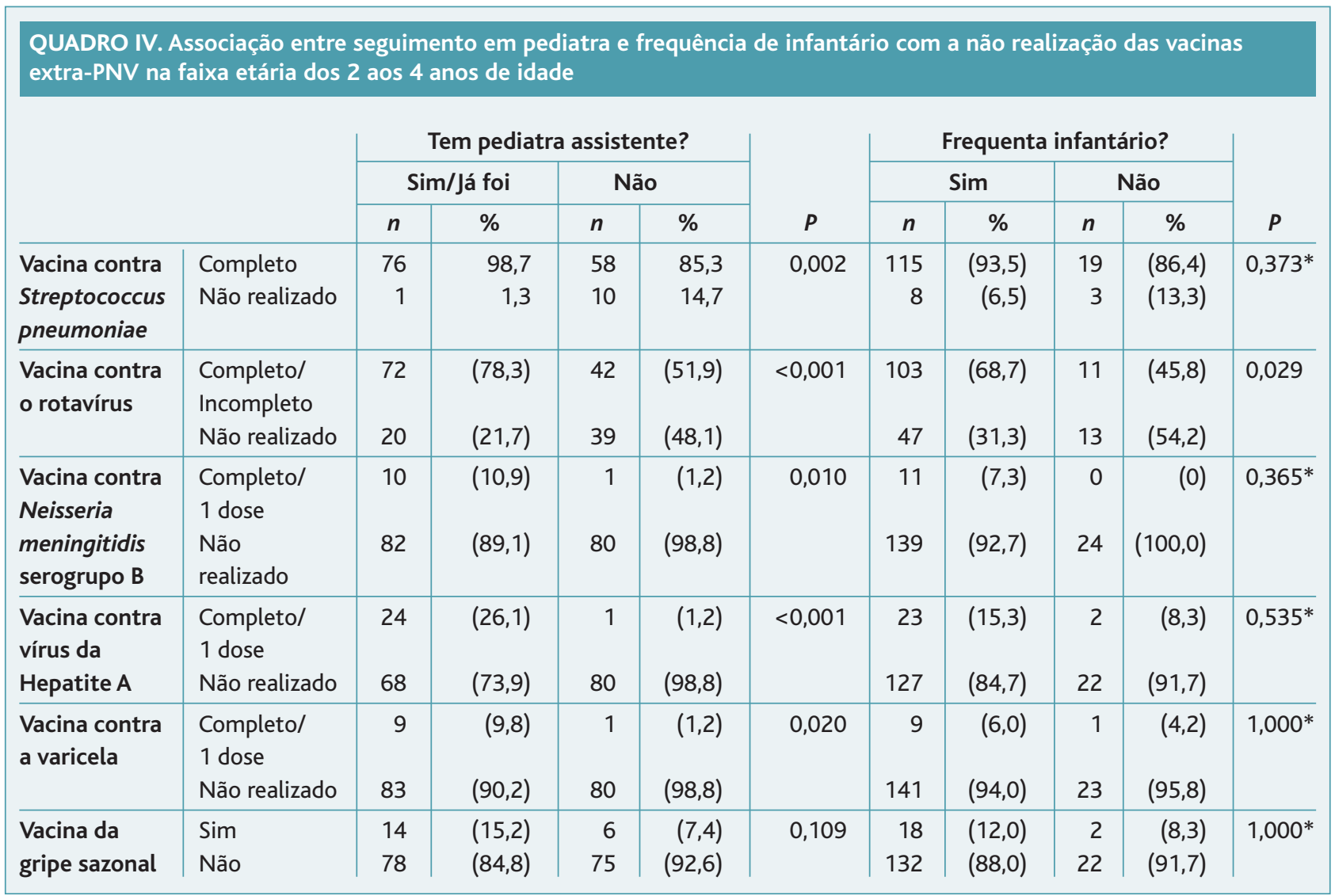

*Resultado do teste exato de Fisher.

que esta é uma vacina que pode ser realizada fora da USF e, portanto, o número de crianças que a realizou pode ser na realidade superior ao apresentado na amostra.

Os resultados deste estudo não são extrapoláveis, atendendo a diversas limitações metodológicas como: ter sido realizado numa USF, numa única região do país, bem como a forma de amostragem utilizada. Uma outra limitação identificada é o facto de apenas terem sido estudados fatores específicos e não ter sido realizada uma pergunta aberta nos questionários telefónicos. Hoje em dia, na nossa sociedade, o facto de os pais não concordarem com a vacinação (em geral ou algumas extra-PNV) pode também ser um fator limitador da vacinação.

No geral, os pediatras são a especialidade que mais tende a recomendar a vacinação extra-PNV aos seus doentes: fazem-no, em média, a cerca de $56,2 \%$ dos seus doentes, enquanto os médicos de MGF admitem fazê lo a 41\%. Desconhecemos o valor atribuído à indústria farmacêutica e à internet, fontes de informação de uso mais discrepante nos dois grupos de profissionais. O preço das vacinas e o facto de considerarem que a vacinação extra-PNV não é adequada a todos os seus doentes são os principais motivos referidos por ambos os grupos de profissionais para nem sempre aconselharem ou nunca aconselharem cada uma das vacinas. Novamente, de acordo com o descrito noutros estudos, surge o preço das vacinas como elemento limitador da prescrição. ${ }^{12-13,19}$ No entanto, o uso de uma amostra de 30 médicos de cada grupo profissional e que globalmente apresentam, em média, menos de 10 anos de prática clínica não nos permite extrapolar resultados, pois desconhecemos, por exemplo, a importância que o maior número de anos de prática clínica poderá ter nas diferentes opiniões. 


\begin{tabular}{|c|c|c|c|c|c|c|c|c|c|c|c|c|c|c|c|}
\hline & & \multicolumn{6}{|c|}{ Escolaridade Mãe } & \multirow{3}{*}{$P$} & \multicolumn{6}{|c|}{ Escolaridade Pai } & \multirow{3}{*}{$P$} \\
\hline & & \multicolumn{2}{|c|}{$\begin{array}{l}\text { Licenciat./ } \\
\text { Bacharelat. }\end{array}$} & \multicolumn{2}{|c|}{$\begin{array}{c}\text { Ensino } \\
\text { secundário }\end{array}$} & \multicolumn{2}{|c|}{$6 .^{\circ}-9 .^{\circ}$ ano } & & \multicolumn{2}{|c|}{$\begin{array}{l}\text { Licenciat./ } \\
\text { Bacharelat. }\end{array}$} & \multicolumn{2}{|c|}{$\begin{array}{c}\text { Ensino } \\
\text { secundário }\end{array}$} & \multicolumn{2}{|c|}{$6 .^{\circ}-9 .^{\circ}$ ano } & \\
\hline & & $n$ & $\%$ & $n$ & $\%$ & $n$ & $\%$ & & $n$ & $\%$ & $n$ & $\%$ & $n$ & $\%$ & \\
\hline $\begin{array}{l}\text { Vacina } \\
\text { contra } \\
\text { Rotavírus }\end{array}$ & $\begin{array}{l}\text { Completo/ } \\
\text { Incompleto } \\
\text { Não } \\
\text { realizado }\end{array}$ & 6 & $\begin{array}{l}(86,0) \\
(14,0)\end{array}$ & $\begin{array}{l}44 \\
15\end{array}$ & $\begin{array}{l}(74,6) \\
(25,4)\end{array}$ & $\begin{array}{l}33 \\
38\end{array}$ & $\begin{array}{l}(46,5) \\
(53,5)\end{array}$ & $<0,001$ & $\begin{array}{r}30 \\
2\end{array}$ & $\begin{array}{r}(93,8) \\
(6,3)\end{array}$ & $\begin{array}{l}21 \\
14\end{array}$ & $\begin{array}{l}(60,0) \\
(40,0)\end{array}$ & $\begin{array}{l}63 \\
43\end{array}$ & $\begin{array}{l}(59,4) \\
(40,6)\end{array}$ & 0,001 \\
\hline $\begin{array}{l}\text { Vacina } \\
\text { contra } \\
\text { Neisseria } \\
\text { meningitidis } \\
\text { serogrupo B }\end{array}$ & $\begin{array}{l}\text { Completo/ } \\
1 \text { dose } \\
\text { Não } \\
\text { realizado }\end{array}$ & 39 & $(9,3)$ & 52 & $(88,1)$ & 71 & $(100,0)$ & - & 29 & $(9,4)$ & 30 & $(85,7)$ & 103 & $(2,8)$ & - \\
\hline $\begin{array}{l}\text { Vacina } \\
\text { contra } \\
\text { vírus da } \\
\text { Hepatite A }\end{array}$ & $\begin{array}{l}\text { Completo/ } \\
1 \text { dose } \\
\text { Não } \\
\text { realizado }\end{array}$ & $\begin{array}{l}13 \\
30\end{array}$ & $\begin{array}{l}(30,2) \\
(69,8)\end{array}$ & $\begin{array}{r}9 \\
50\end{array}$ & $\begin{array}{l}(15,3) \\
(84,7)\end{array}$ & $\begin{array}{r}3 \\
68\end{array}$ & $\begin{array}{r}(4,2) \\
(95,8)\end{array}$ & 0,001 & $\begin{array}{l}14 \\
18\end{array}$ & $\begin{array}{l}(43,8) \\
(56,3)\end{array}$ & $\begin{array}{r}5 \\
30\end{array}$ & $\begin{array}{l}(14,3) \\
(85,7)\end{array}$ & $\begin{array}{r}6 \\
100\end{array}$ & $\begin{array}{r}(5,7) \\
(94,3)\end{array}$ & $<0,001$ \\
\hline $\begin{array}{l}\text { Vacina } \\
\text { contra } \\
\text { varicela }\end{array}$ & $\begin{array}{l}\text { Completo/ } \\
1 \text { dose } \\
\text { Não } \\
\text { realizado }\end{array}$ & $\begin{array}{r}3 \\
40\end{array}$ & $\begin{array}{r}(7,0) \\
(93,0)\end{array}$ & $\begin{array}{r}4 \\
55\end{array}$ & $\begin{array}{r}(6,8) \\
(93,2)\end{array}$ & $\begin{array}{r}3 \\
68\end{array}$ & $\begin{array}{r}(4,2) \\
(95,8)\end{array}$ & - & $\begin{array}{r}3 \\
29\end{array}$ & $\begin{array}{r}(9,4) \\
(90,6)\end{array}$ & $\begin{array}{r}2 \\
33\end{array}$ & $\begin{array}{r}(5,7) \\
(94,3)\end{array}$ & $\begin{array}{r}5 \\
101\end{array}$ & $\begin{array}{r}(4,7) \\
(95,3)\end{array}$ & - \\
\hline $\begin{array}{l}\text { Vacina da } \\
\text { gripe } \\
\text { sazonal }\end{array}$ & $\begin{array}{l}\text { Sim } \\
\text { Não }\end{array}$ & $\begin{array}{r}5 \\
38\end{array}$ & $\begin{array}{l}(11,6) \\
(88,4)\end{array}$ & $\begin{array}{r}9 \\
50\end{array}$ & $\begin{array}{l}(15,3) \\
(84,7)\end{array}$ & $\begin{array}{r}6 \\
65\end{array}$ & $\begin{array}{r}(8,5) \\
(91,5)\end{array}$ & 0,482 & $\begin{array}{r}4 \\
28\end{array}$ & $\begin{array}{l}(12,5) \\
(87,5)\end{array}$ & $\begin{array}{r}8 \\
27\end{array}$ & $\begin{array}{l}(22,9) \\
(77,1)\end{array}$ & $\begin{array}{r}8 \\
98\end{array}$ & $\begin{array}{r}(7,5) \\
(92,5)\end{array}$ & - \\
\hline
\end{tabular}

\section{QUADRO VI. Percentagem média de aconselhamento das vacinas extra-PNV na faixa etária dos 2 aos 4 anos de idade pelos pediatras e médicos de MGF}

\section{Percentagem média}

Em cada 100 crianças, em média a quantas crianças aconselha a vacina contra Streptococcus pneumoniae Em cada 100 crianças, em média a quantas aconselha a vacina contra o rotavírus

Em cada 100 crianças, em média a quantas aconselha a vacina contra Neisseria meningitidis serogrupo B

Em cada 100 crianças, em média a quantas aconselha a vacina contra vírus da hepatite $A$

Em cada 100 crianças, em média a quantas aconselha a vacina contra a varicela

Em cada 100 crianças, em média a quantas aconselha a vacina da gripe sazonal
As vacinas contra Streptococcus pneumoniae, contra o rotavírus e contra a Neisseria meningitidis do serogrupo B são as extra-PNV mais recomendadas no geral (pediatras $82,7 \%$, MGF $69,7 \%$ ), o que reflete a sensibilização de ambos os grupos profissionais para as doenças causadas por estes agentes.

Vários estudos existem acerca da caracterização da vacinação do PNV. No entanto, no que diz respeito à idade pediátrica existem ainda poucos estudos publicados no âmbito da vacinação extra-PNV em Portugal, bem como acerca das posições tomadas por médicos de MGF e pediatras nesta área.

Neste estudo, desemprego e insuficiência económica não se revelaram preponderantes na decisão de não vacinar para a maioria das vacinas. Este facto permite-nos inferir 
que, apesar das condições económicas, os pais colocam a saúde dos seus filhos em primeiro lugar, com tudo o que isso possa implicar.

\section{REFERÊNCIAS BIBLIOGRÁFICAS}

1. Freitas MG. Programa nacional de vacinação e reforma dos cuidados de saúde [The Portuguese national vaccination programme and the reform of primary health care]. Rev Port Clin Geral. 2007;23(4):409-15. Portuguese

2. Nogueira-Silva C, Gonçalves JP. Avaliação da taxa de cobertura vacinal no Centro de Saúde de Braga, nas coortes de nascimento de 1990 a 2005 [Assessment of vaccine coverage of 1990 to 2005 births cohorts, in Braga health center]. Rev Port Clin Geral. 2007;23(5):503-18. Portuguese

3. Cavaco A, Gouveia C, Rodrigues F, Prata F, Varandas L. Recomendações sobre vacinas: actualização 2014. Lisboa: Comissão de Vacinas da Sociedade de Infeciologia Pediátrica e Sociedade Portuguesa de Pediatria; 2014.

4. Direção-Geral da Saúde. Programa nacional de vacinação 2012: norma nº 040/2011, de 21/12/2011, atualização de 26/01/2012. Lisboa: DGS; 2012.

5. Aguiar SI, Brito MJ, Horacio AN, Lopes JP, Ramirez M, Melo-Cristino J. Decreasing incidence and changes in serotype distribution of invasive pneumococcal disease in persons aged under 18 years since introduction of 10-valent and 13-valent conjugate vaccines in Portugal, July 2008 to June 2012. Euro Surveill. 2014;19(12):207-50.

6. Iroh Tam PY, Madoff LC, Coombes B, Pelton SI. Invasive pneumococcal disease after implementation of 13-valent conjugate vaccine. Pediatrics. 2014;134(2):210-7.

7. Instituto Nacional de Estatística. Statistical information [Internet]. Lisbon: INE; [s.d]. Available from: http://www.ine.pt/

8. Miller E, Andrews NJ, Waight PA, Slack MP, George RC. Effectiveness of the new serotypes in the 13-valent pneumococcal conjugate vaccine. Vaccine. 2011;29(49):9127-31.

9. Agócs MM, Serhan F, Yen C, Mwenda JM, Oliveira LH, Teleb N, et al.WHO global rotavirus surveillance network: a strategic review of the first 5 years, 2008-2012. MMWR Morb Mortal Wkly Rep. 2014;63(29):6347.

10. Yih WK, Lieu TA, Kulldorff M, Martin D, McMahill-Walraven CN, Platt R, et al. Intussusception risk after rotavirus vaccination in U.S. infants. N Engl J Med. 2014;370(6):503-12.

11. Moreno-Pérez D, Alvarez García FJ, Arístegui Fernández J, Cilleruelo Ortega MJ, Corretger Rauet JM, García Sánchez N, et al. Vacunación frente al meningococo B: posicionamiento del Comité Asesor de Vacunas de la Asociación Española de Pediatría [Vaccination against meningococcal B disease: public statement of the Advisory Committee on Vaccines of the Spanish Association of Paediatrics (CAV-AEP)]. An Pediatr (Barc). 2015;82(3):198.e1-9. Spanish

12. Davis MM, Ndiaye SM, Freed GL, Kim CS, Clarck SJ. Influence of insu- rance status and vaccine cost on physicians' administration of pneumococcal conjugate vaccine. Pediatrics. 2003;112(3 Pt 1):521-6.

13. Rocha R, Sampaio MJ, Pereira CA, Liberal I. Factores associados ao não cumprimento do Programa Nacional de Vacinação e das vacinas pneumocócica conjugada heptavalente e contra o rotavírus [Factors associated with non compliance with Portuguese National Immunization Program, the heptavalent pneumococcal conjugate vaccine and rotavirus vaccine]. Acta Pediatr Port. 2010;41(5):195-200. Portuguese

14. Gonçalves G, Frutuoso MA, Ferreira MC, Freitas MG. Strategy to increase and access vaccine in the North of Portugal. Euro Surveill. 2005;10(5):98-102.

15. Neves JF, Leça A, Gomes MC, Oliveira M, Ferreira GC. Avaliação do estado vacinal em crianças internadas [Vaccination rates in Lisbon, Portugal]. Nascer Crescer. 2006;15(3):125-8. Portuguese

16. Queirós L, Castro L, Ferreira MC, Gonçalves G. Adesão às novas vacinas conjugadas: vacina anti-meningocócica e anti-pneumocócica [Acceptance of the new conjugate vacines: meningococcal and pneumococcal vaccines, in the cohort born in 1999, in the North Region of Portugal]. Acta Med Port. 2004;17(1):49-53. Portuguese

17. Borrow R. Advances with vaccination against Neisseria meningitidis. Trop Med Int Health. 2012;17(12):1478-91.

18. Sociedade de Infeciologia Pediátrica, Sociedade Portuguesa de Pediatria. Recomendações para a vacinação contra a varicela. Lisboa: SIP; SPP; 2009.

19. Santos H, Pinto E, Valente I, Marinheiro M, Almeida S, Pinto ME. Cobertura vacinal das vacinas meningocócica e pneumocócica num centro de saúde [A survey of meningococcal and pneumococcal vaccination coverage in a primary health care center]. Saúde Infantil. 2008;30(2):62-4.

\section{CONFLITOS DE INTERESSE}

Os autores declaram não possuir qualquer tipo de conflitos de interes

\section{FINANCIAMENTO}

O trabalho relatado neste manuscrito não foi objeto de qualquer tipo de financiamento externo.

\section{COMISSÃO DE ÉTICA}

Estudo realizado após parecer favorável da Comissão de Ética para a Saúde da ARS Norte.

\author{
ENDEREÇO PARA CORRESPONDÊNCIA \\ Joana Teixeira \\ Hospital de Braga \\ Sete Fontes \\ 4710-243 São Victor, Braga \\ E-mail: jiteixeira@hotmail.com
}

Recebido em 09-05-2015

Aceite para publicação em 21-01-2016 


\section{VACCINATION OUTSIDE THE NATIONAL VACCINATION PLAN FOR CHILDREN AGED 2 TO 4 YEARS: FACTORS ASSOCIATED WITH COMPLIANCE FROM THE PERSPECTIVES OF THE PEDIATRICIAN AND THE GENERAL PRACTITIONER}

Aims: We aim to know the factors associated with compliance with recommendations for vaccination of children outside the national vaccination plan among patients of general practitioners (GP) and pediatricians.

Type of Study: Cross-sectional study.

Local: Ruães Family Health Unit (Cávado I Group of Health Centres).

Population: Children aged 2-4 years old and pediatricians and GP from Braga.

Methods: Electronic health records of children were studied. A questionnaire was administered by telephone interview of parents or guardians from August 2014 to January 2015. We used an electronic questionnaire with online response by GP and pediatricians to evaluate GP and pediatricians' compliance with recommendations. Data were analyzed using the Statistical Package for Social Sciences (SPSS, Chicago, IL, USA), version 17.0 for Microsoft Windows ${ }^{\circledR}$.

Results: We selected a sample of 180 children, aged of 2-4 years old. Vaccination coverage against Streptococcus pneumoniae was $81.7 \%$. There was no statistically significant association between unemployment of the parent or guardian and non-compliance vaccines outside the plan. We found higher rates of vaccination against Neisseria meningitidis serogroup $B$ and against hepatitis A virus (HAV) in the absence of economic difficulties ( $p$ value of Fisher $=0.008$ and $p=0.046$, respectively). Association between rotavirus vaccination and kindergarten attendance was statistically significant $(p=0.029)$. There was also a statistically significant association between university-level education of the father or mother and rotavirus and hepatitis A vaccination. Vaccines outside the national plan were recommended by the family doctor in $46 \%$ of cases. Vaccination with vaccines outside the national plan was higher in children who were or had been followed by pediatrician (except flu vaccine).

The sample of $30 \mathrm{GP}$ and 30 pediatricians revealed that the most commonly recommended vaccine was that against Streptococcus pneumoniae. The order of importance attributed to vaccines was the same in both groups of professionals. The main reason for not recommending the Streptococcus pneumoniae vaccine was its price.

Discussion and conclusions: For most vaccines, unemployment and economic difficulties are not decisive factors in the decision not to vaccinate. This emphasizes the parents' role in vaccination. Measures are required to ensure a fair access to different vaccine options.

Keywords: Vaccination; Extra-Portuguese Immunization Program; Noncompliance. 


\section{ANEXO I \\ Guião telefónico | Questionário aplicado a pais ou encarregados de educação}

Duração $=2 \min$

"Bom dia. Eu sou o/a (...) da USF Ruães, e trabalho com o seu (sua) médico de família. Estou a falar com o Sr. (a) Gostaria de informar o Sr. (a) de que está a ser realizado na sua USF um estudo que pretende avaliar os fatores associados à realização das vacinas extra-PNV nas crianças dos 2-4 anos de idade. Assim sendo, solicitamos a sua colaboração neste estudo. A sua participação é voluntária e confidencial. Aceita participar no estudo? (...) Muito obrigado! Peço-lhe então que responda às seguintes questões que fazem parte do questionário, está bem? Agradeço-lhe o tempo dispensado e sua colaboração."

Perguntas do questionário (...)

\section{Caracterização da criança e do agregado familiar}

\begin{tabular}{l|l}
\hline Idade & "Quantos anos tem a criança?" ou "Qual a data de nascimento?" \\
\hline Género & "O seu filho é um menino ou uma menina?" \\
\hline Tamanho do agregado familiar & $\begin{array}{c}\text { "Quantas crianças e quantos adultos constituem o agregado familiar?" ou } \\
\text { "Quantas crianças e quantos adultos moram em casa?" }\end{array}$ \\
\hline Escolaridade dos pais & "Qual o nível de escolaridade da mãe? E do pai?" \\
\hline Idade dos pais & "Qual a idade da mãe? E do pai?" \\
\hline Pais desempregados & "Atualmente está alguém desempregado? A mãe está desempregada? E o pai?" \\
\hline Seguimento e Vacinação & "O seu filho é seguido no pediatra?" \\
\hline Pediatra assistente & "O seu filho já frequenta infantário ou creche?" \\
\hline Infantário & "Sabe dizer-me quem o informou e esclareceu acerca das vacinas extra-PNV?" \\
\hline Esclarecimento acerca das vacinas extra-PNV
\end{tabular}




\section{ANEXO II \\ Questionário aplicado a médicos}

Exmo(a) Colega,

Encontramo-nos a realizar um estudo acerca da vacinação extra-PNV, perspetiva do pediatra versus médico assistente e fatores associados à não realização.

Neste sentido, agradecemos a sua colaboração dedicando três minutos ao preenchimento do questionário, que se encontra disponível para resposta até 30/Novembro.

Garantimos que a informação recolhida é estritamente confidencial e anónima e não será tratada em termos individuais. O seu endereço de e-mail foi utilizado apenas para esta mensagem e não voltará a ser usado nem terá qualquer ligação com o que responder no questionário.

Convidamo-lo(a) a participar no preenchimento do questionário, através do seguinte link:

\section{I - Vacinação extra-PNV dos 2-4 anos: perspetiva do pediatra vs médico de família}

1. Coloque, por ordem decrescente de importância (do mais importante para o menos importante), a sua opinião relativamente às vacinas extra-PNV nas crianças até aos 4 anos de idade.

- Vacina contra Streptococcus pneumoniae

- Vacina antirrotavírus

- Vacina contra Neisseria meningitidis serogrupo B

- Vacina contra VHA

- Vacina contra a varicela

- Vacina da gripe sazonal

2. Qual ou quais considera as suas principais fontes de informação relativamente às vacinas extra-PNV?

- Colegas

- Literatura/revistas médicas

- Internet

- Indústria farmacêutica

- Congressos e reuniões científicas

- DGS

- SPP

- Outras fontes de informação

2.1 Se outro, qual?

3. Em cada 100 crianças, em média a quantas aconselha a tomar:

- Vacina contra Streptococcus pneumoniae

- Vacina antirrotavírus

- Vacina contra Neisseria meningitidis serogrupo B

- Vacina contra VHA

- Vacina contra a varicela

- Vacina da gripe sazonal
4. Para cada uma das vacinas, escolha qual ou quais os motivos pelos quais nem sempre a aconselha:

Vacina contra Streptococcus pneumoniae

- Custo/preço

- Doença benigna que não justifica a vacina

- Não adequado a todos os doentes

- Acho que os pais do doente iam recusar

- Falta de informação/experiência

- Vacina controversa/polémica

- Não vejo necessidade

- Vejo a criança pela primeira vez já fora do grupo etário

- Efeitos secundários/Fatores de risco

- Não se aplica

Vacina antirrotavírus

- Custo/preço

- Doença benigna que não justifica a vacina

- Não adequado a todos os doentes

- Acho que os pais do doente iam recusar

- Falta de informação/experiência

- Vacina controversa/polémica

- Não vejo necessidade

- Vejo a criança pela primeira vez já fora do grupo etário

- Efeitos secundários/Fatores de risco

- Não se aplica

Vacina contra Neisseria meningitidis serogrupo B

- Custo/preço

- Doença benigna que não justifica a vacina

- Não adequado a todos os doentes

- Acho que os pais do doente iam recusar

- Falta de informação/experiência

- Vacina controversa/polémica 
- Não vejo necessidade

- Vejo a criança pela primeira vez já fora do grupo etário

- Efeitos secundários/Fatores de risco

- Não se aplica

Vacina contra VHA

- Custo/preço

- Doença benigna que não justifica a vacina

- Não adequado a todos os doentes

- Acho que os pais do doente iam recusar

- Falta de informação/experiência

- Vacina controversa/polémica

- Não vejo necessidade

- Vejo a criança pela primeira vez já fora do grupo etário

- Efeitos secundários/Fatores de risco

- Não se aplica

Vacina contra a varicela

- Custo/preço

- Doença benigna que não justifica a vacina

- Não adequado a todos os doentes

\section{II - Informações gerais}

1. Anos de prática clínica:

2. Especialidade a que pertence:

- Pediatria

- Medicina Geral e Familiar

Esta ligação está associada apenas a este questionário e ao seu endereço de correio eletrónico. Não reencaminhe esta mensagem.
- Acho que os pais do doente iam recusar

- Falta de informação/experiência

- Vacina controversa/polémica

- Não vejo necessidade

- Vejo a criança pela primeira vez já fora do grupo etário

- Efeitos secundários/Fatores de risco

- Não se aplica

Vacina da gripe sazonal

- Custo/preço

- Doença benigna que não justifica a vacina

- Não adequado a todos os doentes

- Acho que os pais do doente iam recusar

- Falta de informação/experiência

- Vacina controversa/polémica

- Não vejo necessidade

- Vejo a criança pela primeira vez já fora do grupo etário

- Efeitos secundários/Fatores de risco

- Não se aplica 